\title{
Effect of peripheral blood T-lymphocyte subpopulation test on gastric cancer monitoring and prognostic evaluation.
}

\author{
Ting Lv', Cai-Yun Zhang ${ }^{2}$, Lei Dong ${ }^{3}$, He-Ping Wu ${ }^{\text {* }}$ \\ ${ }^{1}$ Department of Gastroenterology, Nuclear Industry 215 Hospital of Shaanxi Province, Xianyang, Shaanxi, PR China \\ ${ }^{2}$ Department of Neurology, Nuclear Industry 215 Hospital of Shaanxi Province, Xianyang, Shaanxi, PR China \\ ${ }^{3}$ Department of Gastroenterology, the Second Affiliated Hospital of Xi'an Jiaotong University, Xi'an, Shaanxi, PR China
}

\begin{abstract}
Objective: This paper aims to discuss the effect of peripheral blood T-lymphocyte subpopulation test on gastric cancer monitoring and prognostic evaluation.

Methods: Ninety-three patients with gastric cancer were recruited for this prospective study and randomly divided into two groups. The conventional treatment group was treated with DDP+5trihydroxypyrimidine, and the observation group was treated with combined raltitrexed and oxaliplatin. Venous blood samples were collected on an empty stomach before and after the treatment. The proportions of Th1 and Th2 were determined by flow cytometry analysis. Serum levels of IL-2 and IL-10 were tested by an enzyme-linked immunosorbent assay kit. Another 33 volunteer patients were recruited in the same period for the control group.

Results: Before the treatment, the conventional treatment group and the observation group possessed higher proportions of Th1 and Th2 and serum levels of IL-2 and IL-10 but significantly lower Th1/Th2 and IL-2/IL-10 than the control group $(P<0.05)$. However, the difference between the conventional treatment group and the observation group was not statistically significant $(P>0.05)$. After the treatment, the observation group exhibited lower proportions of Th1 and Th2 and serum levels of IL-2 and IL-10 but higher Th1/Th2 and IL-2/IL-10 than the conventional treatment group $(\mathrm{P}<0.05)$.

Conclusions: Peripheral blood T-lymphocyte subpopulation test can be used as an index for gastric cancer monitoring and prognostic evaluation and merits clinical promotion.
\end{abstract}

Keywords: Raltitrexed, Oxaliplatin, Gastric cancer, Th1/Th2D.

Accepted on October 24, 2017

\section{Introduction}

With rapid social and economic development and the accelerated pace of life, gastric cancer morbidity has soared as a result of increasing poor habits in daily activities and diet [1]. Gastric cancer exhibits the highest death rate among all malignant tumors. According to statistical data, 90\% of new cancer cases in China are advanced gastric carcinoma, and only $30 \%$ of the patients survived for $5 \mathrm{y}$ after the radical operation. Most patients died of tumor relapse and metastasis [2,3]. Tlymphocyte subpopulation plays an important role in the incidence and development of gastric cancer. In this paper, 80 patients with gastric cancer admitted in our hospital from September 2014 to September 2016 were selected for Tlymphocyte subpopulation detection.

\section{Data and Methods}

\section{Clinical data}

A total of 93 patients diagnosed with gastric cancer by cytological or pathological tissue examination were recruited. In addition, 33 healthy volunteers subjected to physical examination in the same period were included as the control group. The 93 patients were divided using a random number table into conventional treatment group $(n=46)$ and observation group $(n=47)$. The conventional treatment group included 29 males and 17 females aged 36-75 y (average: $52.3 \pm 6.85 \mathrm{y}$ ). The observation group included 27 males and 20 females aged 33-73 y (average: $52.5 \pm 7.03 \mathrm{y}$ ). The control group included 19 males and 14 females aged 31-70 y (average: $52.0 \pm 6.33$ y). No statistically significant difference was noted among the three groups in terms of age and gender $(\mathrm{P}>0.05)$. 


\section{Treatments}

The conventional treatment included DDP +5 trihydroxypyrimidine: DDP20 $\mathrm{mg} \bullet \mathrm{m}^{2}$ given by intravenous drip for $1-5 \mathrm{~d}$ and 5 -FU500 $\mathrm{mg} \bullet \mathrm{m}^{-2}$. The intravenous drip was maintained for $24 \mathrm{~h}$ for $1-5 \mathrm{~d}$ and repeated every $4 \mathrm{w}$. The observation group was given L-OHP130 $\mathrm{mg}^{\circ} \mathrm{m}^{2}$ by intravenous drip for $2 \mathrm{~h}$ at $1 \mathrm{~d}$ and $\mathrm{s}-140 \mathrm{mg} \cdot \mathrm{m}^{-2}$ twice a day at 1-14 d after breakfast and dinner. The treatment cycle was repeated every $3 \mathrm{w}$, and the clinical effect and toxicity were evaluated every $2 \mathrm{w}$. The treatment period lasted for $28 \mathrm{~d}$.

\section{Test of indices}

For the control group, elbow venous blood samples were collected upon hospital admission for physical examination. For the observation group, elbow venous blood samples (3-5 $\mathrm{ml}$ ) were collected $1 \mathrm{~d}$ before and within $5 \mathrm{~d}$ of the treatment. The samples were stored in two tubes. Tube A was used to test Th1/Th2 by flow cytometry analysis. Fluorescent-labeled antibodies CD3-PerCP, IFN-r-FITC, and IL-4-PE were provided by $\mathrm{BD}$ Company (USA). Tube $\mathrm{B}$ was used to determine the serum concentration of Th1 cytokines (IL-2) and Th2 cytokines (IL-10) by fully automatic biochemical analysis and an Enzyme-Linked Immunosorbent Assay (ELISA) kit (Beijing Equation Biotech Co., Ltd.). The procedures were conducted in strict compliance with the specifications.

\section{Statistical analysis}

SPSS19.0 was used for data analysis. Enumeration data were expressed in constituent ratio or percentage and were verified by $\chi^{2}$-test. Quantitative data were expressed in $\overline{\mathrm{x}} \pm \mathrm{S}$. Variance analysis was conducted for multigroup comparison. Data with statistically significant differences were verified by LSD-t-test. Intergroup comparison was verified by t-test. Differences at $\mathrm{P}<0.05$ were considered statistically significant.

\section{Results}

\section{Th1/Th2}

Before the treatment, the proportions of Th1 and Th2 were higher, whereas the Th1/Th2 was lower in the conventional treatment and observation groups than those in the control group $(\mathrm{P}<0.05)$. However, the difference between the conventional treatment and observation groups was not statistically significant $(\mathrm{P}>0.05)$. After the treatment, the proportions of Th1 and Th2 were lower, whereas the Th1/Th2 was higher in the observation group than those in the conventional treatment group $(\mathrm{P}<0.05)$ (Table 1).

\section{IL-2/IL-10}

Before the treatment, the IL-2 and IL-10 levels were higher in the conventional treatment and observation groups than those in the control group $(\mathrm{P}<0.05)$. However, the difference between the conventional treatment group and the observation group was not statistically significant $(\mathrm{P}>0.05)$. After the treatment, the IL-2 and IL-10 levels were lower, but the IL-2/IL-10 was higher in the observation group than those in the conventional treatment group $(\mathrm{P}<0.05)$ (Table 2$)$.

Table 1. Comparison of Th1/Th2 among all groups before and after the treatment $(\bar{x} \pm S)$. Note: LSD-t1, P1: conventional treatment group versus control group; LSD-t2, P2: Observation group versus control group; LSD-t3, P3: conventional treatment group versus observation group.

\begin{tabular}{|c|c|c|c|c|c|c|c|c|c|}
\hline Item & Time & $\begin{array}{l}\text { Control } \\
(n=33)\end{array}$ & group & $\begin{array}{l}\text { Conventional treatment } \\
\text { group }(n=46)\end{array}$ & $\begin{array}{l}\text { Observation } \\
(n=47)\end{array}$ & group & LSD-t1, P1 & LSD-t2, P2 & LSD-t3, P3 \\
\hline \multirow[t]{3}{*}{ Th1 (\%) } & Before & $16.5 \pm 1.05$ & & $23.4 \pm 2.83$ & $23.7 \pm 2.65$ & & $13.34,<0.05$ & $14.79,<0.05$ & $0.528,>0.05$ \\
\hline & After & I & & $17.4 \pm 2.03$ & $15.3 \pm 1.67$ & & $2.330,<0.05$ & $3.649,<0.05$ & $5.453,<0.05$ \\
\hline & $t, P$ & I & & $11.68,<0.05$ & $18.39,<0.05$ & & & & \\
\hline \multirow[t]{3}{*}{ Th2 (\%) } & Before & $2.33 \pm 0.52$ & & $4.01 \pm 0.62$ & $4.15 \pm 0.74$ & & $5.744,<0.05$ & $12.17,<0.05$ & $0.988,>0.05$ \\
\hline & After & I & & $2.75 \pm 0.41$ & $2.26 \pm 0.35$ & & $4.035,<0.05$ & $0.720,>0.05$ & $6.203,<0.05$ \\
\hline & $t, P$ & I & & $11.50,<0.05$ & $15.83,<0.05$ & & & & \\
\hline \multirow[t]{3}{*}{ Th1/Th2 } & Before & $7.08 \pm 1.02$ & & $5.84 \pm 0.66$ & $5.71 \pm 0.63$ & & 6.603 & $7.420,<0.05$ & $0.972,>0.05$ \\
\hline & After & I & & $6.32 \pm 0.35$ & $6.77 \pm 0.43$ & & 4.737 & $1.865,<0.05$ & $5.528,<0.05$ \\
\hline & $t, P$ & I & & $4.458,<0.05$ & $9.527,<0.05$ & & & & \\
\hline
\end{tabular}

Table 2. Comparison of IL-2/IL-10 among all groups before and after the treatment $(\bar{x} \pm S)$. Note: LSD-t1, P1: conventional treatment group versus control group; LSD-t2, P2: Observation group versus control group; LSD-t3, P3: conventional treatment group versus observation group.

\begin{tabular}{llll}
\hline Item Time & $\begin{array}{l}\text { Control } \\
(n=33)\end{array}$ & group & $\begin{array}{l}\text { Conventional } \\
\text { group }(n=46)\end{array}$
\end{tabular} treatment $\begin{aligned} & \text { Observation } \\
& (n=47)\end{aligned}$ group LSD-t1,P1 LSD-t3,P3




\begin{tabular}{llllllll}
\hline IL-2 & Before & $16.3 \pm 2.35$ & $83.2 \pm 5.33$ & $85.1 \pm 6.02$ & $67.54,<0.05$ & $62.31,<0.05$ & $1.620,>0.05$ \\
\cline { 2 - 7 } & After & $/$ & $38.4 \pm 4.57$ & $31.5 \pm 4.08$ & $37.58,<0.05$ & $7.086,<0.05$ & $7.778,<0.05$ \\
\cline { 2 - 7 } & t, P & $/$ & $43.28,<0.05$ & $50.53,<0.05$ & & $49.69,<0.05$ & $0.588,>0.05$ \\
\hline IL-10 & Before & $13.5 \pm 2.11$ & $75.3 \pm 6.32$ & $74.8 \pm 6.85$ & $54.01,<0.05$ & $10.95,<0.05$ & $12.18,<0.05$ \\
\cline { 2 - 7 } & After & $/$ & $31.5 \pm 4.02$ & $21.6 \pm 3.86$ & $23.52,<0.05$ & $2.465,<0.05$ & $1.150,>0.05$ \\
\cline { 2 - 7 } & t, P & $/$ & $39.66,<0.05$ & $46.39,<0.05$ & & $3.395,<0.05$ & $7.988,<0.05$ \\
\hline
\end{tabular}

\section{Discussion}

Patients with gastric cancer present a highly complicated immune dysfunction. On the one hand, the functions of innate immunocytes, such as mononuclear macrophages, neutrophils granulocytes, and dendritic cells, change [4]. The first line of defense against microbial infection is destroyed, and the decreased body resistance to pathogenic bacteria is aggravated. On the other hand, the proportion of the T-lymphocyte subpopulation and the contents of cytokines are altered. The most typical modification is the imbalance between antiinflammatory and proinflammatory responses. Raltitrexed is a cytotoxic anticancer drug that synthesizes a series of polyglutamic acid compounds with high cytotoxicity by inhibiting thymidylate synthase and dihydrofolate reductase [5]. These polyglutamic acid compounds remain in the cells for long periods to simultaneously inhibit DNA and develop cytotoxicity. Therefore, dosage selection can directly influence the safety of the therapy. Oxaliplatin is a newly developed third-generation platinum drug that promotes tumor cell apoptosis by inhibiting DNA synthesis [6]. This drug involves different drug-resistance mechanisms and slightly inhibits bone marrow. Oxaliplatin has no renal toxicity and is thus relatively safe for use. Modern pharmacological studies have proven that oxaliplatin not only inhibits histiocytic hydrolysis but also affects immunity regulation. However, the underlying immune mechanism remains unknown.

Among T-lymphocytes, Th1 and Th2 are important subpopulations that regulate the proinflammatory-antiinflammatory balance. In this study, patients with gastric cancer possessed higher proportions of Th1 and Th2 and slightly higher Th1/Th2 than the control group. Consistent with our findings, reports stated that Th1 mainly participates in cell immune responses and delays hypersensitive inflammatory responses. The T-cell subpopulation can excrete proinflammatory cytokines, such as interferon gamma (IFN- $\gamma$ ), IL-2, and tumor necrosis factor alpha, and can thus intensify inflammatory responses [7]. Th2 mainly excretes antiinflammatory cytokines, such as IL-4 and IL-10, thereby inhibiting inflammatory responses to some extent. Moreover, Th2 can impede immunity by decreasing the activity of antigen-presenting cells and Th1. A previous study performed a quantitative analysis on IL-2 and IL-10 and reported that their serum levels are basically related to the distribution trends of Th1 and Th2. This result proves that the serious proinflammatory and anti-inflammatory responses in patients with gastric cancer are accompanied by skewed Th1/Th2 to Th2 [8]. In general, the pathological state will present as immunity inhibition.

After the treatment, Th1/Th2 and IL-2/IL10 in the conventional treatment group and observation group are corrected to some extent, especially in the latter. Previous studies demonstrated that severe proinflammatory cytokine infiltration into the blood causes general inflammatory responses and cell apoptosis. This condition is an important pathological feature of gastric cancer and a key factor that accelerates its advancement. Extensive research has shown that the immune mechanism of patients with gastric cancer is more complicated than usually known and is a dynamic process [9]. In the early stage, considerable excretion of proinflammatory medium and the activation of inflammatory responses are the main pathological features; these phenomena are followed by a compensatory increase in the excreted amount of antiinflammatory media. Immunity inhibition dominated by Th2 cell reaction occurs in the late period, and the body becomes increasingly sensitive to pathogens. Therefore, the different quantitative research conclusions on Th1 and Th2 may be due to the simultaneous rise of $\mathrm{Th} 1$ and $\mathrm{Th} 2$ cell responses. Raltitrexed and oxaliplatin combined therapy can enhance immune function and relieve the inflammatory responses of patients through multiple means. For example, reports proved that the combined raltitrexed and oxaliplatin can increase the number of inductive T-cell/inhibitive T-cells and enhance the general immune function of patients to resist immunity inhibition [10]. In the present study, raltitrexed and oxaliplatin combined therapy adjusted the quantities and function of Th1/Th2 in patients with gastric cancer. This mode of action may be a component of the anti-inflammatory mechanism of the therapy.

\section{Conclusion}

A Th1/Th2 drift occurs in patients with gastric cancer. Raltitrexed and oxaliplatin combined therapy can adjust the 
quantities and function of $\mathrm{Th} 1 / \mathrm{Th} 2$ to maintain the balance between proinflammatory and anti-inflammatory responses. As a result, the body damage from inflammatory responses is reduced, and disease development is halted. The T-lymphocyte subpopulation test can be used as a reference for gastric cancer monitoring and prognostic evaluation and hence merits clinical promotion.

\section{References}

1. Olsson J, Wikby A, Johansson B, Lofgren S, Nilsson BO, Ferguson FG. Age-related change in peripheral blood Tlymphocyte subpopulations and cytomegalovirus infection in the very old: the Swedish longitudinal OCTO immune study. Mech Ageing Dev 2000; 121: 187-201.

2. Guo N, Lin G. Effects of nonsurgical periodontal therapy on serum inflammatory factor levels in patients with chronic kidney disease and periodontitis. Biomed Res India 2017; 28: 3899-3902

3. Zhao C, Zhang Y, Yu Z. Inhalable chitosan coated solid lipid nanoparticles for interventional delivery to lung cancer. Lat Am J Pharm 2017; 36: 12-18.

4. Wiegers GJ, Croiset G, Reul JM, Holsboer F, de Kloet ER. Differential effects of corticosteroids on rat peripheral blood T-lymphocyte mitogenesis in vivo and in vitro. Am J Physiol 1993; 265: 825-830.

5. Rajkumar K, Swaminathan P. Optimized energy aware scheduling to minimize makespan in distributed systems. Biomed Res India 2017; 28: 2877-2883.
6. Heimdal JH, Aarstad HJ, Olofsson J. Peripheral blood Tlymphocyte and monocyte function and survival in patients with head and neck carcinoma. Laryngoscope 2000; 110: 402-407.

7. Covas MI, Esquerda A , García-Rico A, Mahy N. Peripheral blood T-lymphocyte subsets in autoimmune thyroid disease. J Invest Allerg Clin 1992; 2: 131-135.

8. Miloglu FD, Senol O, Kadioglu Y, Bilici M. Determination of retinol and beta-carotene after beta-carotene administration in patients with stomach cancer via HPLCDAD method in human plasma. Lat Am J Pharm 2017; 36: 740-749.

9. Desrues B, Delaval P, Genetet N, Pencole C, Merdrignac G, Dassonville J, LHuillier JP, Bourguet P, Kernec J. Does peripheral blood T-lymphocyte population distribution in sarcoidosis provide a prognostic clue? Sarcoidosis 1991; 8: 129-133.

10. Duan X, Wu Q, Zhu X, Shi Y. Improving sleep quality relieves occupational stress in nurses of cardiac surgical intensive care unit. Biomed Res India 2017; 28: 3934-3940.

\section{*Correspondence to}

He-Ping $\mathrm{Wu}$

Department of Gastroenterology

Nuclear Industry 215 Hosptial of Shaanxi Province

PR China 